\title{
Staphylococcal Toxic Shock Syndrome Caused By An Intravaginal Product. A Case Report
}

\author{
Monica Marton* \\ Swedish Medical Center, Department of Anesthesiology and Critical Care, Seattle, USA
}

\begin{abstract}
Staphylococcal toxic shock syndrome (STSS) represents a potentially lethal disease, and survival depends primarily on the early initiation of appropriate treatment. As the clinical picture at presentation is usually common, frequently this could lead to misdiagnosis and delays in the initiation of the proper therapy. The case of a 43-years old female who developed a staphylococcal septic shock syndrome caused by a forgotten intravaginal tampon is reported.
\end{abstract}

Keywords: Staphylococcus aureus, toxic shock, endotoxin

Received: 25 September 2015 / Accepted: 15 December 2015

\section{INTRODUCTION}

Staphylococcal toxic shock syndrome (STSS) represents a potentially lethal disease, and the survival depends primarily on the early initiation of appropriate treatment [1].

The asymptomatic presence of Staphylococcus aureus (SA) in places such as the nasopharyngeal or perineal area is recorded in a large percent of the population [1]. However, in certain conditions, this bacteria could produce a severe form of STSS, and the use of intravaginal tampons is one of the factors described as increasing the risk of developing STSS in patients with SA bloodstream infection [2]. Systemic effects of the SA are caused by the exotoxin produced by the germ and released in the systemic circulation, being responsible for multi-organ system failure and toxic shock. As the clinical picture, at presentation, is usually common, consisting of vomiting, confusion and fever, this frequently results in a misdiagnosis and delay in the initiation of the proper therapy. The case of a 43-years old female who developed a staphylococcal septic shock syndrome caused by a forgotten intravaginal tampon is detailed.

\section{CASE REPORT}

A 43-years old female presented to the emergency unit with fever $\left(101.7^{\circ} \mathrm{F}\right)$, vomiting and diffuse abdominal pain of unknown aetiology. The symptoms had manifested themselves suddenly, eighteen hours before presentation, without being preceded by any prodromes, and had persisted since then. At presentation, the patient had a normal blood pressure $(127 / 78 \mathrm{~mm}$ $\mathrm{Hg}$ ), tachycardia, with a heart rate of 100 beats/minute, looked anxious and reported feeling weak.

The patient declined any possible pregnancy, stating that menses had started three days before presentation, denied any use of antibiotics in the last six months and admitted a recent history of intravenous drug usage and iv heroin administration within forty-eight hours. More detailed information about the patient's history was difficult to obtain due to an associated encephalopathy that had developed during the current disorder.

At presentation, the patient was somnolent, without any nuchal rigidity, exhibiting a mild scleral icterus, injection marks in the right antecubital area and palpable right axillary adenopathy. Lung auscultation revealed a mild expiratory wheezing. Laboratory results are presented in Table 1, indicating normal values for white blood count, positive tests for opiates and cannabis, low levels of fibrinogen and slightly altered values of coagulation parameters. Stool cultures showed the presence of parasites and Clostridium difficile.

According to the clinical status and laboratory results, a suspicion of pyelonephritis was raised and the patient was admitted to a medical unit, where antibiotic treatment was initiated, consisting in the administration of two grams of Rocephin. 
At twelve hours after admission, the patient developed a deterioration of her haemodynamic status with low blood pressure (96/40 $\mathrm{mm} \mathrm{Hg}$ ), tachycardia (110 beats per minute), and laboratory parameters which indicated hepatic failure and an increase in lactate levels (Table 1). As the patient status continued to deteriorate, she was transferred to the intensive care unit (ICU) where a peripherally inserted central catheter (PICC line) was introduced, and volemic resuscitation was initiated. Chest X-ray, head computed tomography, abdominal ultrasonography and pelvic ultrasonography were all normal, and Doppler ultrasound of the inferior limbs indicated no sign of deep venous thrombosis or septic thrombophlebitis.

At that stage, the differential diagnoses were: acute pyelonephritis, endocarditis, septic shock of unknown origin, leptospirosis or tick-borne disease. However, no clear diagnostic criteria were present for any of these illnesses. As the patient developed, in the same day, an abdominal rash, other rare aetiologies were also evaluated, such as Kawasaki disease, Reye syndrome, erythema multiforma or Rocky Mountain spotted fever. No clear evidence for any of these was documented.

After several hours, despite intravenous antibiotic therapy, the patient presented with persistent fever $\left(103-104^{0} \mathrm{~F}\right)$, moderate pleural effusion, excessive amount of fluid surrounding the liver and thickened walled gallbladder, with massive accumulation of pericholecystic fluid. Transthoracic echocardiography indicated the presence of an echogenic mass in the right atrium, which on transesophageal examination proved to be a non-vegetative Chiari network. The patient developed severe bradyarrhythmia and self-limited ventricular tachycardia which responded to therapy. As the patient's status continued to deteriorate and the patient remained febrile despite antibiotic therapy, a re-

Table 1. Biochemistry results at presentation and in evolution

\begin{tabular}{lcc} 
& At presentation & At $\mathbf{1 2}$ hours \\
\hline WBC $\left(\times 10^{3} / \mu \mathrm{L}\right)$ & 6200 & 7.000 \\
\hline Lactate $(\mathrm{mg} / \mathrm{dL})$ & 0.8 & 2.4 \\
\hline INR & 1.4 & 1.8 \\
\hline PT $(\mathrm{sec})$ & 16.4 & 17 \\
\hline Fibrinogen $(\mathrm{mg} / \mathrm{dL})$ & 160 & 140 \\
\hline ALAT $(\mathrm{IU} / \mathrm{L})$ & 120 & 255 \\
\hline ASAT $(\mathrm{IU} / \mathrm{L})$ & 145 & 243 \\
\hline CK-MB $(\mathrm{IU} / \mathrm{L})$ & 872 & 1490 \\
\hline
\end{tabular}

peated infectious workup was indicated. This consisted of repeated blood cultures, stool studies and insertion of a straight urinary catheter for the collection of sterile urine samples. During the bladder catheterization, a retained vaginal tampon, overlooked for more than thirty hours, was discovered.

The patient was diagnosed with STSS, caused by the intravaginal product, the tampon being immediately removed. The appropriate modification of antibiotic therapy was initiated, with the intravenous administration of Clindamycin 900 mg every 8 hours, Vancomycin 1 gram every twelve hours and Gentamycin $40 \mathrm{mg}$ every twelve hours, for five days, followed by the oral administration of Clindamycin $150 \mathrm{mg}$ every six hours and Doxicilin $100 \mathrm{mg}$ every twelve hours, for fourteen days. On the removal of the tampon and commencement of a new antibiotic regimen, the patient's status significantly improved within twelve hours, followed by the rash disappearing and normalization of biochemistry parameters.

\section{Discussions}

Staphylococcus aureus (SA) is a gram-positive coccus, which produces local invasion and tissue destruction, leading to severe forms of impetigo, cellulitis and endocarditis. From the epidemiological point of view, Staphylococcus aureus is a commensal in humans, the latter being, in many cases, asymptomatic carriers, the reservoir of germs being located in the nasopharynx and the rectum. Studies indicated the perineal presence of Staphylococcus aureus, in over $90 \%$ of women with STSS $[3,4]$. Cutaneous colonization with this germ is usually brief and repetitive, and transmission is from person to person. The carrier rate of Staphylococcus aureus was reported in $20 \%$ of the general population, $75 \%$ of haemodialysis patients, and $40 \%$ of intravenous drugs users [5]. Asymptomatic SA nasal carriage was identified in $38 \%$ of patients on chronic haemodialysis and in $18 \%$ of medical personnel [6].

Toxic-shock syndrome caused by Staphylococcus aureus was first described in 1978, in a case series of seven children who presented with fever, rash and vomiting which progressed to acute renal failure, hepatic dysfunction and severe prolonged shock [7]. At that time, the systemic effects of SA were attributed to the exotoxin produced by this organism, as the SA has been isolated from the mucosal or abscess sites without being identified in the systemic circulation. STSS 
is now considered to be a toxin-mediated multisystem disease, presenting initially with non-specific symptoms, rapidly worsening and leading, in most severe cases, to multiorgan failure and death [8].

SA produces a variety of toxins, which may be the reason for the higher rate of its resistance in the community, and allow dissemination of the organism within the community [9]. One of the toxins produced by SA is an enterotoxin with superantigenic and emetic effects [10] and which was identified as the toxin responsible for the vomiting symptoms in these cases.

In a recently published prospective cohort study, infective endocarditis represented the most frequent complications in patients with SA bloodstream infection, being associated with a high mortality rate [11]. In another study, $29.8 \%$ of patients with SA bloodstream infection developed severe sepsis and 12.9\% developed septic shock, with a $36 \%$ mortality at ninety days [12].

Published data indicates that in the ICU, the energy deficit produced by the bloodstream infections with methicillin-resistant types of SA is significantly higher than the one caused by other frequently identified germs [13], especially in prolonged mechanically ventilated patients, suggesting that SA produces a more severe form of infection than other ICU-acquired bloodstream infections.

The risk factors for the development of STSS include usage of intravaginal tampons, previous STSS, postpartum or postabortion SA infection, septorhinoplasty or surgical wounds. The presence of intravaginal or intrauterine devices has been described as a cause of fatal STSS [2]. The development of STTS in a patient using a menstrual cup for the first time has been described, with similarities to the present report [14]. In males, SA has been reported as an important cause of prostatic abscesses, some resulting in death [15].

The most common clinical presentation of patients with STSS includes fever, tachycardia, hypotension and confusion. SA is also responsible for several cutaneous manifestations, from rash to fulminant purpura, resulting mainly from the effect of staphylococcal exotoxin and being associated, in most cases, with the toxic shock syndrome [16].

A recent study indicated a mortality of $16.4 \%$ in patients with SA bloodstream infection, with mortality reaching $40-50 \%$, in cases of STTS [1]. The risk factors for mortality in patients with SA bloodstream infections were identified as old age, the longer retainment of central venous catheters, prosthetic heart valves, severe sepsis, septic shock, APACHE II score and fever persisting for forty-eight hours after diagnosis [1]. Maintenance of a peripherally inserted central catheter has been identified as one of the best predictors of complicated SA bloodstream infections especially in immunocompromised patients, cancer patients with central-line catheters, and SA infections [17].

The STSS is related to the production of the staphylococcal exotoxin, which has been identified in over $90 \%$ of menstrual-related cases and in $40-60 \%$ of nonmenstrual related cases [18]. The production of exotoxin 1 is facilitated by a neutral vaginal $\mathrm{pH}$ and by the presence of tampon-containing synthetic fibers.

The enterotoxins produced by the SA, together with toxin 1, lead to a nonspecific T-lymphocyte stimulation without normal antigenic recognition, producing a massive production of cytokines that are involved in the pathogenesis of the severe shock, via the release of IL-1, IL-2, TNF and interferon. At the same time, the enterotoxins act as powerful super-antigens, producing mast cell activation that is responsible for the development of skin rash, or severe illnesses such as severe pneumonia [19]. The super-antigen-mediated cytokine release leads to respiratory failure, renal failure, vascular leakage and toxic shock, being the main responsible for the STSS [20], and superantigen genes were more common identified in menstrual than in non-menstrual fluids (89\% vs. 50\%) [21]. TNF-alfa and interleukins are responsible for the development of arterial hypotension, shock, renal failure, hepatic failure and death. One of the superantigens produced by the SA is the TSS toxin-1, responsible for almost of all cases of TSS cases in the USA, and together with enterotoxin $\mathrm{B}$ and $\mathrm{C}$ responsible for most cases of non-menstrual TSS [22].

In most of the cases, a host immune response plays a significant role in the pathogenesis of the disease, as patients who develop STSS are frequently unable to produce antibodies, leading to frequent relapses. A unique form of adaptive immune suppression has been described as a result of the interaction between SA super-antigens and the immune system of the host, immunosuppression directly linked with the poor survival of these patients [22].

Particular aspects of the present case are represented by the co-existence of more predisposing factors for septic shock and a complex clinical presentation, that could easily lead to diagnostic errors. The patient presented a recent history of intravenous drug adminis- 
tration, associated with an echocardiographic image of an echogenic mass in the right atrium. The Chiari network, in the right atrium, could have been confused with vegetation on the tricuspid valve, in the context of a clinical picture characteristic of infective endocarditis. Patients with drug abuse, are frequently immunocompromised, and the incidence and the severity of SA infections are higher in severely diseased, immunocompromised patients. For instance, analysis of the large EPIC II database, including thirteen patients from 1256 ICUs, indicated the presence of methicillinresistant SA to a significantly greater extent in cirrhotic patients compared to non-cirrhotic ones [23]. At the same time, the patient had a documented infection with Clostridium difficile, as shown by stool analysis, an infection that could also be extremely severe in immunocompromised patients. The clinical picture at presentation, including vomiting and nausea in the presence of a gastrointestinal infection, could also represent another source of misdiagnosis.

\section{CONCLUSION}

This case raises awareness of the importance of complete clinical examination in patients presenting with typical symptoms of unknown aetiology.

\section{CONFLICT OF INTEREST}

The author declares that she has no conflict of interest.

\section{REFERENCES}

1. De Rosa FG, Corcione S, Motta I, et al. Risk factors for mortality in patients with Staphylococcus aureus bloodstream infection. J Chemother. 2015:1973947815Y0000000076. [Epub ahead of print]

2. Klug $C D$, Keay $C R$, Ginde AA. Fatal toxic shock syndrome from an intrauterine device. Ann Emerg Med. 2009;54:701-3.

3. David M, Daum R. Community-Associated MethicillinResistant Staphylococcus aureus: Epidemiology and Clinical Consequences of an Emerging Epidemic. Clin Microbiol Rev. 2010;23:616-87.

4. Stingley RL, Liu H, Mullis LB, Elkins CA, Hart ME. Staphylococcus aureus toxic shock syndrome toxin-1 (TSST-1) production and Lactobacillus species growth in a defined medium simulating vaginal secretions. J Microbiol Methods. 2014;106:57-66.

5. Kluytmans J, van Belkum A, Verbrugh $\mathrm{H}$. Nasal carriage of Staphylococcus aureus: epidemiology, underlying mechanisms, and associated risks. Clin Microbiol Rev. 1997;10:505-20.
6. Diawara I, Bekhti K, Elhabchi D, et al. Staphylococcus aureus nasal carriage in hemodialysis centers of Fez, Morocco. Iran J Microbiol. 2014;6:175-83.

7. Todd J, Fishaut M, Kapral F, Welch T. Toxic-shock syndrome associated with phage-group-I Staphylococci. Lancet. 1978;2:1116-8.

8. Chan Y, Selvaratnam V, Garg N. Toxic shock syndrome post open reduction and Kirschner wire fixation of a humeral lateral condyle fracture. BMJ Case Rep. 2015;2015. pii: bcr2015210090.

9. Jiang W, Zhou Z, Zhang K, Yu Y. Epidemiological investigation of community-acquired Staphylococcus aureus infection. Genet Mol Res. 2013;12:6923-30.

10. Ono HK, Sato'O Y, Narita K, et al. Identification and Characterization of a Novel Staphylococcal Emetic Toxin. Appl Environ Microbiol. 2015;81:7034-40.

11. Le Moing V, Alla F, Doco-Lecompte T, et al. Staphylococcus aureus Bloodstream Infection and Endocarditis- A Prospective Cohort Study. PLoS One. 2015;10:e0127385.

12. Paulsen J, Mehl A, A, Solligard E, Asvold BO, Damas JK. Epidemiology and outcome of Staphylococcus aureus bloodstream infection and sepsis in a Norwegian county 19962011: an observational study. BMC Infect Dis. 2015;15:116.

13. Ekpe K, Novara A, Mainardi JL, Fagon JY, Faisy C. Methicillinresistant Staphylococcus aureus bloodstream infections are associated with a higher energy deficit than other ICU-acquired bacteremia. Intensive Care Med. 2014;40:1878-87.

14. Mitchell MA, Bisch S, Arntfield S, Hosseini-Moghaddam SM. A confirmed case of toxic shock syndrome associated with the use of a menstrual cup. Can J Infect Dis Med Microbiol. 2015;26:218-20.

15. Lachant DJ, Apostolakos M, Pietropaoli A. Methicillin Resistant Staphylococcus aureus Prostatic Abscess with Bacteremia. Case Rep Infect Dis. 2013;2013:613961.

16. Honarpisheh H, Camp R, Lazova R. Staphylococcal Purpura Fulminans: Report of a Case. Am J Dermatopathol. 2015;37:6436.

17. El Zakhem A, Chaftari AM, Bahu R, et al. Central line-associated bloodstream infections caused by Staphylococcus aureus in cancer patients: Clinical outcome and management. Ann Med. 2014;46:163-8.

18. Dinges MM, Orwin P, Schlievert P. Exotoxins of Staphyloccocus aureus. Clin Microbiol Rev. 2000;13:16-34.

19. Tilahun AY, Karau M, Ballard A, et al. The impact of Staphylococcus aureus-associated molecular patterns on staphylococcal superantigen-induced toxic shock syndrome and pneumonia. Mediators Inflamm. 2014;2014:468285.

20. Low DE. Toxic shock syndrome: major advances in pathogenesis, but not treatment. Crit Care Clin. 2013;29:651-75.

21. DeVries AS, Lesher L, Schlievert PM, et al. Staphylococcal toxic shock syndrome 2000-2006: epidemiology, clinical features, and molecular characteristics. PLoS One. 2011;6:e22997. 
Available online at: www.jccm.ro

22. Kulhankova K, King J, Salgado-Pabón W. Staphylococcal toxic shock syndrome: superantigen-mediated enhancement of endotoxin shock and adaptive immune suppression. Immunol Res. 2014;59:182-7.
The Journal of Critical Care Medicine 2016;2(1) • 55

23. Gustot T, Felleiter P, Pickkers P, et al. Impact of infection on the prognosis of critically ill cirrhotic patients: results from a large worldwide study. Liver Int. 2014;34:1496-503. 